\title{
Severe Cushing's syndrome and bilateral pulmonary nodules: beyond ectopic ACTH
}

\author{
Carlos Tavares Bello', Emma van der Poest Clement ${ }^{2}$ and Richard Feelders ${ }^{3}$ \\ ${ }^{1}$ Endocrinology Department, Hospital de Egas Moniz, Centro Hospitalar de Lisboa Ocidental, Lisboa, Portugal, \\ 2Faculty of Medicine, Erasmus University, Rotterdam, The Netherlands, and ${ }^{3}$ Endocrinology Department, \\ Erasmus MC, Rotterdam, The Netherlands
}

Correspondence should be addressed to C Tavares Bello Email bello.carlos04@gmail.com

\section{Summary}

Cushing's syndrome is a rare disease that results from prolonged exposure to supraphysiological levels of glucocorticoids. Severe and rapidly progressive cases are often, but not exclusively, attributable to ectopic ACTH secretion. Extreme hypercortisolism usually has florid metabolic consequences and is associated with an increased infectious and thrombotic risk. The authors report on a case of a 51-year-old male that presented with severe Cushing's syndrome secondary to an ACTH-secreting pituitary macroadenoma, whose diagnostic workup was affected by concurrent subclinical multifocal pulmonary infectious nodules. The case is noteworthy for the atypically severe presentation of Cushing's disease, and it should remind the clinician of the possible infectious and thrombotic complications associated with Cushing's syndrome.

\section{Learning points:}

- Severe Cushing's syndrome is not always caused by ectopic ACTH secretion.

- Hypercortisolism is a state of immunosuppression, being associated with an increased risk for opportunistic infections.

- Infectious pulmonary infiltrates may lead to imaging diagnostic dilemmas when investigating a suspected ectopic ACTH secretion.

- Cushing's syndrome carries an increased thromboembolic risk that may even persist after successful surgical management.

- Antibiotic and venous thromboembolism prophylaxis should be considered in every patient with severe Cushing's syndrome.

\section{Background}

Endogenous Cushing's syndrome (CS) is a rare disease with an estimated incidence of 0.7-2.4 per million population per year (1). Clinical presentation varies according to the severity of biochemical hypercortisolism and its underlying cause. ACTH-dependent causes are responsible for $80 \%$ of all CS cases and among these, ACTH-secreting pituitary adenomas (Cushing's disease) and ectopic ACTH secretion (EAS) are the most frequent underlying causes (80\% and 20\%, respectively) (2).
Severe CS is often attributable to EAS by a malignant neoplasm (small-cell carcinoma of the lung, pulmonary and pancreatic neuroendocrine tumours, medullary thyroid carcinoma, among others). Hyperglycaemia, hypokalaemia, hypertension, altered mental status, venous thromboembolism and systemic infections are more common in EAS and have been positively correlated with the degree of hypercortisolaemia (3). In severe cases, apart from treating hypercortisolism itself, 
management of associated comorbidities/consequences, namely opportunistic infections and thromboembolic complications, is of utmost importance. The authors report on a case of a 51-year-old male with severe CS, in whom diagnostic investigations were influenced by a severe concomitant infection, and the final outcome was determined by a thrombotic event.

\section{Case presentation}

A 51-year-old male, with an unremarkable medical history, was admitted to the hospital for a new onset right-sided ptosis and diplopia. The patient had no other subjective complaints, although his family members described a 3-month history of abnormal social behaviour, paranoid ideations and altered mental status. The complaints had had a sudden onset and were rapidly worsening. Upon admission, physical examination revealed hypertension (blood pressure of $180 / 110 \mathrm{mmHg}$ ), hyperglycaemia $(8.2 \mathrm{mmol} / \mathrm{L})$, no fever and an apparently adequate behaviour. Patient also evidenced a moon face, skin hyperpigmentation, abdominal obesity, proximal muscle weakness and bilateral ankle pitting oedema. Furthermore, eye examination revealed bilateral chemosis and rightsided oculomotor palsy (ptosis, miosis and diplopia in all directions). No funduscopic evidence of optic nerve compression was present.

During admission, the patient's condition worsened from both the clinical (psychosis) and laboratory

Table 1 Initial laboratory investigation.

\begin{tabular}{l}
\hline Laboratory parameter \\
\hline Haemoglobin $(\mathrm{mmol} / \mathrm{L})$ \\
Leucocyte count $\left(\times 10^{9}\right)$ \\
Platelet count $\left(\times 10^{9}\right)$ \\
CRP (mg/dL) \\
Fasting blood glucose (mmol/L) \\
Potassium (mmol/L) \\
Corrected calcium (mmol/L) \\
Creatinine (mg/dL) \\
Morning plasma cortisol (nmol/L) \\
ACTH (ng/L) \\
Urinary free cortisol (24h) \\
FSH (U/L) \\
LH (U/L) \\
Total testosterone (nmol/L) \\
Prolactin (U/L) \\
IGF-1 (nmol/L) \\
TSH (IU/L) \\
Free thyroxine (pmol/L)
\end{tabular}

\begin{tabular}{cccc} 
Result & & Reference range \\
\cline { 1 - 1 } 7.8 & & $8.6-10.5$ \\
11.1 & & $3.5-10$ \\
104 & & $150-370$ \\
1.5 & & $0-9$ & \\
12.8 & & $4-6.1$ \\
2.3 & & $3.5-5.1$ \\
2.12 & & $2.2-2.65$ \\
71 & & $65-115$ \\
1355 & & $200-700$ \\
58.4 & & $0-11$ \\
3106 & & $5-133$ \\
0.1 & & $2-7$ \\
$<0.1$ & & $1.5-8$ \\
1.44 & & $10-30$ \\
0.4 & & $<0.36$ \\
8.2 & & $7.9-28.1$ \\
0.28 & & $0.4-4.3$ \\
6.2 & & $11-25$ \\
& &
\end{tabular}

$\mathrm{ACTH}$, Adreocorticotrophic hormone; CRP, $\mathrm{C}$ reactive protein; $\mathrm{FSH}$, Follicular stimulating hormone; IFG-1, Insulin-like growth factor 1; LH, Luteinzing hormone; TSH, Thyrotropin. standpoints, thus prompting for complex medical management strategies.

\section{Investigation}

Results of any relevant tests that were carried out, in particular those influencing decisions on patient management.

Admission laboratory investigation was remarkable for leucocytosis, thrombocytopenia, hypokalaemia, elevated levels of fasting plasma cortisol, 24-h free urinary cortisol and ACTH, hypogonadotrophic hypogonadism and central hypothyroidism (Table 1).

Overnight $1 \mathrm{mg}$ dexamethasone suppression test was compatible with autonomous cortisol secretion (postdexamethasone morning plasma cortisol of $1454 \mathrm{nmol} / \mathrm{L}$ ).

Because of the probable underlying hypopituitarism, and new-onset oculomotor palsy, the patient underwent a magnetic resonance imaging (MRI) of the sellar region that revealed an invasive pituitary macroadenoma with cavernous sinus invasion (Fig. 1). Considering the acute presentation of severe ACTH-dependent hypercortisolism, cross-sectional CT imaging of chest and abdomen was performed to detect a possible ectopic source of ACTH production, evidencing a solid lesion of the right upper pulmonary lobe with $16 \times 14 \mathrm{~mm}$, along with multiple smaller solid images on both lung fields (Fig. 2). Both the larger and the multiple smaller solid pulmonary lesions were 18F-FDG-PET positive (Fig. 3).

In the first week after admission, CRP levels rose to a maximum of $39 \mathrm{mg} / \mathrm{L}$. Cultures and serological tests did not reveal mycobacterial, typical and atypical bacterial, parasitic and viral infections. Bronchoalveolar lavage was negative for both malignant cells and infectious agents. Opportunistic agents, namely Pneumocystis jirovecii and Aspergillus, were also not identified. Although CT-guided lung lesion biopsy was performed, the yielded material was not enough for the establishment of a definite diagnosis.

The differential diagnosis then included a corticotroph macroadenoma with pulmonary infection and an ACTH producing lung tumour with a pituitary metastasis.

\section{Treatment}

Initial medical therapy included: glucocorticoid receptor antagonist, Mifepristone $200 \mathrm{mg} 3$ times daily, to counteract glucocorticoid excess; antipsychotics and benzodiazepines for the psychotic manifestations; oral and intravenous high doses of potassium supplements for 


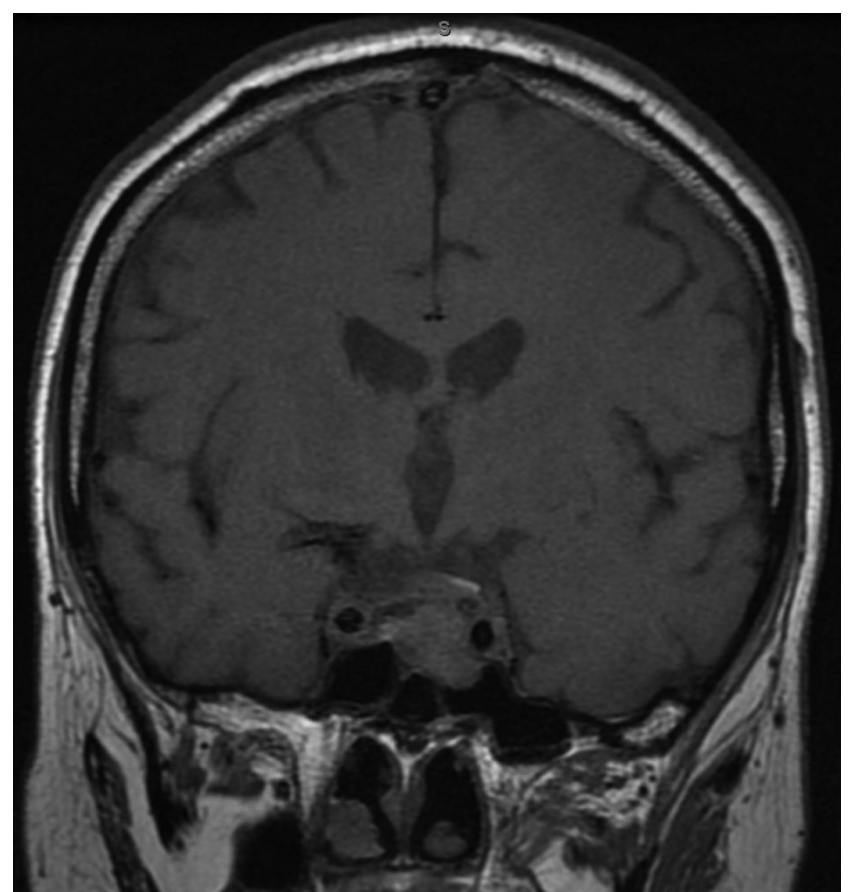

Figure 1

Cranial MR - sellar mass with suprasellar extension and cavernous sinus invasion.

the severe refractory hypokalaemia; spironolactone for arterial hypertension and hypokalaemia; subcutaneous insulin protocol for hyperglycaemia; co-trimoxazol as Pneumocystis prophylaxis; and therapeutical doses of low-molecular-weight heparin (Fraxiparine) for venous thromboembolism prophylaxis. Aggressive medical treatment ( $400 \mathrm{mg}$ spironolactone/day, $240 \mathrm{mmol}$ of i.v. potassium chloride per day and high dose antipsychotics) led to a biochemical stabilisation, despite worsening of psychotic symptoms.

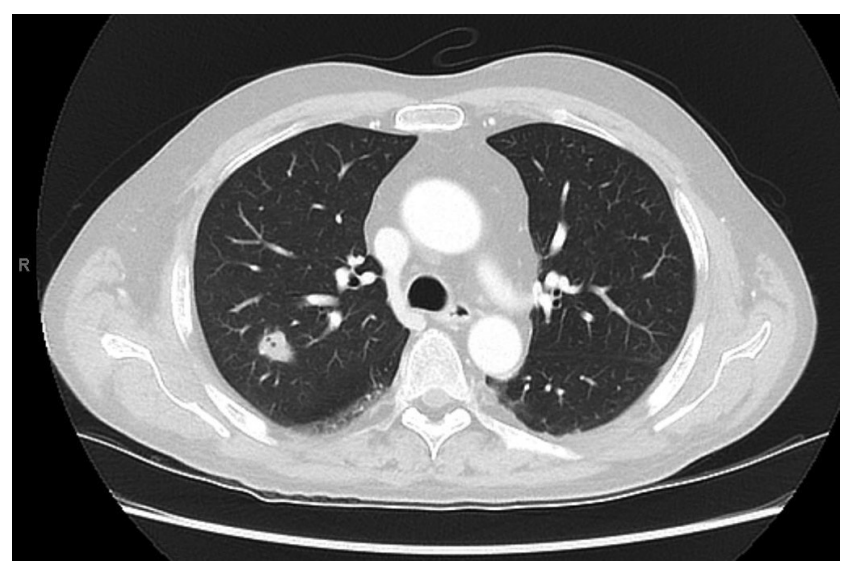

Figure 2

Pulmonary CT - solid mass on the right upper lobe $(16 \times 14 \mathrm{~mm})$.

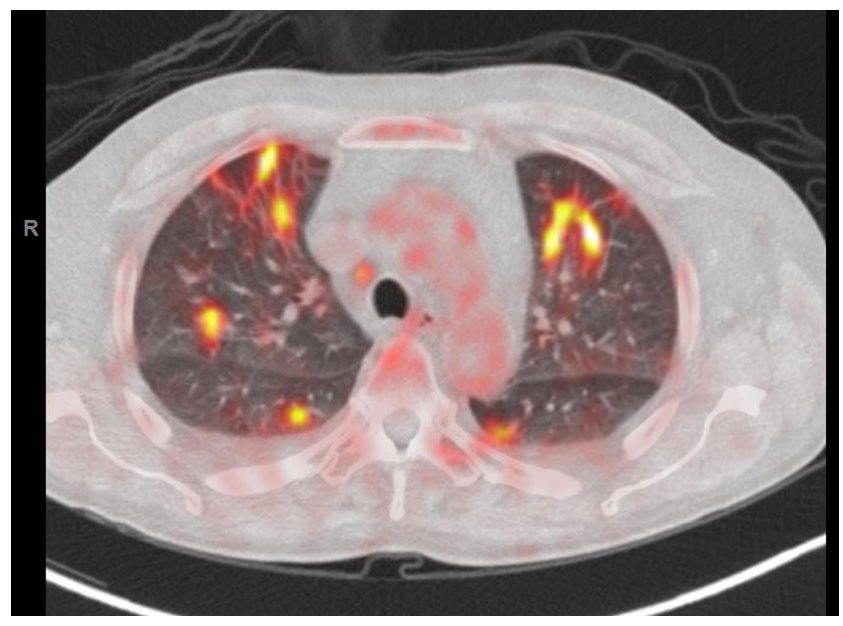

Figure 3

18F-FDG-PET-CT of the lungs - positive uptake in many lung segments possibly suggesting a multifocal neoplasm.

The severe and rapidly progressive clinical picture, that was refractory to medical therapy, warranted an urgent definitive therapy. Therefore, a laparoscopic bilateral adrenalectomy was performed with immediate biochemical control.

The procedure was uneventful, except for a postoperative hospital-acquired pneumonia that was successfully treated with broad-spectrum antibiotics (piperacillin-tazobactam and ciprofloxacin followed by levofloxacin monotherapy). After the procedure, the patient gradually improved regarding hypertension, hyperglycaemia and psychotic symptoms.

One month after adrenalectomy, the invasive pituitary macroadenoma was transsphenoidally resected. Post-operative pituitary MRI showed an intrasellar tumour remnant (Fig. 4) that demonstrated significant growth over a period of 3 months. Subsequently, the patient underwent pituitary radiotherapy (30 fractions - total radiation dose of $57 \mathrm{~Gy}$ ) resulting in a small decrease in tumour volume.

\section{Outcome and follow-up}

Clinical status improved soon after bilateral adrenalectomy with respect to behaviour, hyperglycaemia, hypertension and hypokalaemia. Pituitary adenomectomy led to a gradual improvement in oculomotor palsies, with ptosis resolving in approximately 3 weeks and diplopia over 2 months.

Adrenal and pituitary pathology reports described diffuse adrenal hyperplasia and pituitary apoplexy respectively. No data regarding MIB index were available from the pituitary specimen. The non-apoplectic pituitary 
adenoma tissue sample stained positive for ACTH and was negative for GH, FSH, LH, TSH and prolactin.

After both surgical procedures, low-molecularweight heparin was stopped. Pneumocystis prophylaxis (co-trimoxazole) was maintained for 3 months after CS diagnosis, being discontinued after sustained negative microbiological investigations and resolution of hypercortisolism.

Pulmonary lesions completely regressed after the employed antibiotic strategies, discarding the diagnosis of lung malignancy (Fig. 5).

Maintenance medication included hydrocortisone, fludrocortisone, testosterone gel, levothyroxine, amlodipine and pantoprazole. Clinical condition remained stable for 16 months, without any clinical or radiological evidence of disease progression. At the 16th month of follow-up, the patient presented with extensive bilateral cerebral venous thrombosis of the sigmoid and transverse sinus as well as of the internal jugular veins for which anticoagulant therapy was started. This was complicated by a symptomatic increased intracranial pressure for which the patient was treated by external drainage followed by placement of a ventriculoperitoneal shunt, which was complicated by pneumonia for which mechanical ventilation was needed. During this admission, the patient developed an intraventricular and intraparenchymal bleeding of which he ultimately died.

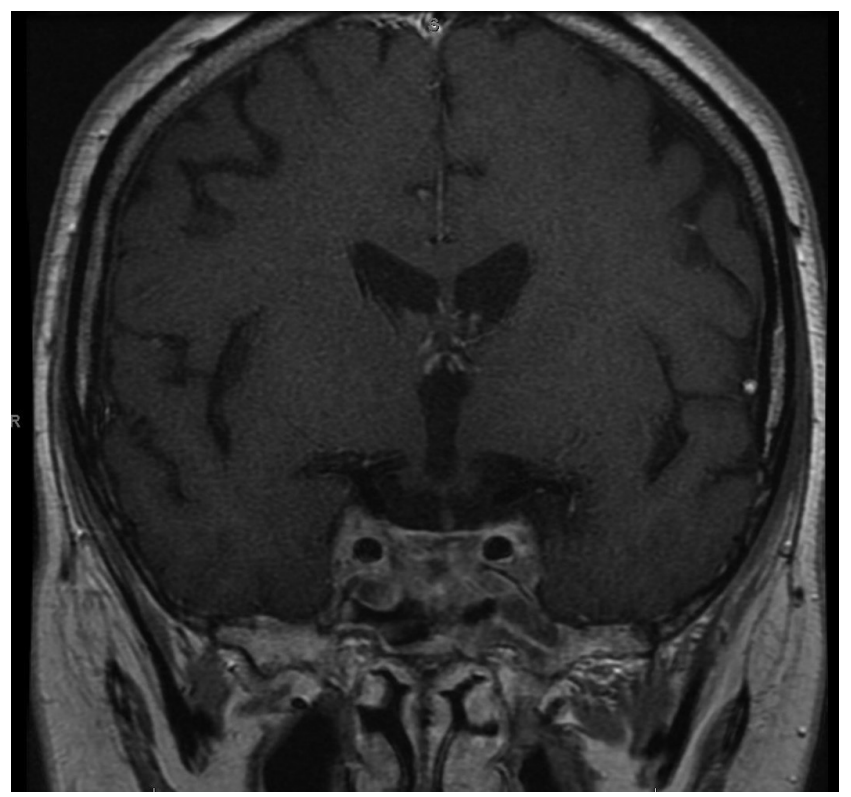

Figure 4

First MRI after pituitary surgery ( 2 weeks) demonstrating a reduction in lesion size with the resulting tumour remnant.

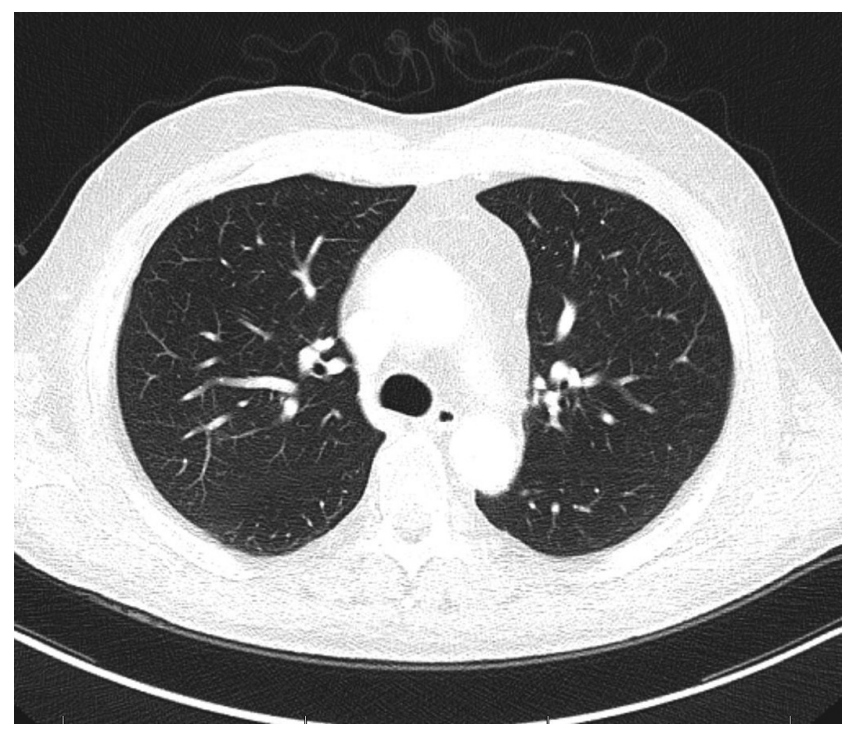

Figure 5

Pulmonary CT 2 months after the diagnosis and antibiotic treatment.

\section{Discussion}

CS has a broad spectrum of clinical manifestations and clinical course. Severe and rapidly progressive cases are often caused by EAS from an underlying malignant neoplasm such as small-cell carcinoma or a neuroendocrine tumour of the lung (4). Cushing's disease (CD), clinical course of which is typically more indolent, may occasionally also have a florid presentation, such as that described in the present case report. Nevertheless, when faced with very severe ACTH-dependent hypercortisolism, one should consider ectopic ACTH as the most probable cause and pursue rapid biochemical control and timely etiological diagnosis.

As pituitary adenomas are thought to affect $10.6 \%$ of the general population, many patients with CS may actually harbour a non-functioning pituitary adenoma. Furthermore, up to $12 \%$ of patients with EAS have pituitary MRI abnormalities. In order to minimise diagnostic inaccuracies, recent guidelines have suggested a $6 \mathrm{~mm}$ pituitary adenoma diameter cut-off for considering the adenoma as an obvious cause for the $\mathrm{CD}(5,6,7)$. The distinction between CD and EAS usually relies on bilateral inferior petrosal sinus venous sampling (BIPSS). For the diagnosis of CD, BIPSS has a sensitivity of $96 \%$ and specificity of $100 \%$ when the central to peripheral ratios of basal and CRH-stimulated serum ACTH gradients are greater than 2 and 3 respectively (8).

Our patient's case evoked an important diagnostic dilemma. On the one hand, the patient's clinical and biochemical presentation, along with the multiple 
pulmonary PET-positive solid lesions were highly suggestive of EAS and small-cell carcinoma of the lung. In addition, sellar metastases are often accompanied by ophthalmic cranial nerve palsies. On the other hand, the new-onset ptosis due to an underlying invasive pituitary adenoma along with the elevated inflammatory markers might otherwise suggest aggressive CD complicated by a pulmonary infection.

Severe CS is a medical emergency that is associated with life-threatening complications (infectious, thromboembolic, cardiovascular or psychiatric). In such cases, prompt control of hypercortisolism should be urgently pursued, even if the diagnostic investigation is incomplete (7). Medical treatment is the first-line treatment and includes steroidogenesis inhibitors (ketoconazole, metyrapone, etomidate, mitotane), glucocorticoid receptor antagonists (mifepristone) and pituitary targeting drugs (pasireotide, cabergoline). In severe CS, metyrapone, ketoconazole and mifepristone are often preferred based on their quick onset of action and efficacy. Depending on treatment response and clinical status, bilateral adrenalectomy should be considered, as it provides immediate hormonal control (7). This was the rationale underlying management decisions on our patient.

Besides treatment of hypercortisolism, optimal CS care includes the simultaneous management of diseaserelated complications, namely mineralocorticoid effects of cortisol excess, infections and thromboembolism.

When severe, hypercortisolism leads to hypokalaemic metabolic alkalosis, hypertension and oedema. The cortisol excess saturates type 2 11-betahydroxysteroid dehydrogenase enzyme in the distal nephron that converts biologically active cortisol into inactive cortisone. As a result, there is mineralocorticoid receptor overstimulation by cortisol. The ensuing hypokalaemia and hypertension are deleterious, favouring the occurrence of cardiac arrhythmias and cardiovascular events, making their treatment of utmostimportance. Mineralocorticoid receptor antagonists (such as spironolactone and eplerenone) in high doses are effective agents in reversing hypokalaemia and reducing peripheral oedema. Regarding blood pressure control, these agents have modest effects; therefore, patients often require additional antihypertensive agents. Glucocorticoid level normalisation is the most effective therapeutic measure (9).

Hypercortisolism is associated with a state of immunosuppression. By affecting both the innate and adaptive immune system, CS predisposes patients to bacterial, viral, fungal and parasitic infections (10).
Furthermore, clinical manifestations of infections may be scarce, since the body may be unable to mount an adequate immune response, becoming sepsis clinically evident only after effective cortisol-lowering therapy (11). Our patient had no symptoms or signs of respiratory tract infection or sepsis, despite having multiple foci of pulmonary infection and leucocytosis. As such, antibiotic prophylaxis regarding Pneumocystis infection is recommended in patients with severe hypercortisolism (urinary free cortisol above 5 times the upper limit of normal) (7).

Thromboembolic disease is also more common in CS than in the general population. Venous thromboembolism not provoked by surgical intervention is estimated to occur in up to $2.5 \%$ of CS patients. Current evidence indicates that the hypercoagulable state in CS is attributed to both activation of the coagulation cascade with cortisol-induced elevations of von Willebrand factor, fibrinogen, Factor VIII and IX and an impaired fibrinolytic activity (12). Additionally, thrombotic diathesis may persist after surgery, the risk being highest within the first 4 post-operative weeks. CS treatment guidelines suggest thromboprophylaxis perioperatively in all patients and the need for prolonged treatment should be individualised based on patients' individual risk factor profile. It should further be noted that the coagulation profile in CS patients may remain altered up to one year after surgical cure (7).

Our patient's case illustrates many of the challenges posed to clinicians managing complex CS patients. Firstly, CD can present with severe hypercortisolism, mimicking the clinical picture of EAS. Secondly, CS is associated with an increased risk for infectious complications and antibiotic prophylaxis is warranted in severe cases. Additionally, CT-suspicious, PET-positive pulmonary infiltrates in patients with a severe hypercortisolism might be infectious in nature warranting formal microbiological studies in addition to primary tumour investigation. Lastly, thromboembolism prophylaxis should also be considered beyond the perioperative period, especially in high-risk patients, since coagulation abnormalities may persist well beyond successful disease management.

\section{Declaration of interest}

The authors declare that there is no conflict of interest that could be perceived as prejudicing the impartiality of the research reported.

\section{Funding}

This research did not receive any specific grant from any funding agency in the public, commercial or not-for-profit sector. 


\section{Patient consent}

Consent could not be obtained because the patient is deceased and his next of kin were not reachable.

\section{Author contribution statement}

Carlos Tavares Bello: article writing and revision; Emma van der Poest Clement: article revision; Richard Feelders: attending physician and article revision.

\section{References}

1 Susmeeta TS, Lynnette KN \& Richard AF 2015 Cushing's syndrome: epidemiology and developments in disease management Clinical Epidemiology 7 281-293. (doi:10.2147/CLEP.S44336)

2 André L, Richard AF, Constantine AS \& Lynnette KN 2015 Cushing's syndrome. Lancet 386(9996) 913-927. (doi:10.1016/S01406736(14)61375-1)

3 David JT, Nancy M, Ioannis I \& Lynnette N 2002 Association of hypertension and hypokalemia with cushing's syndrome caused by ectopic ACTH secretion: a series of 58 cases. Annals of the New York Academy of Sciences 970 134-144. (doi:10.1111/j.1749-6632.2002. tb04419.x)

4 Andrea MI \& Andrea L 2007 Ectopic ACTH syndrome. Arquivos Brasileiros de Endocrinologia and Metabologia 51 1217-1225.
5 Hall WA, Luciano MG, Doppman JL, Patronas NJ \& Oldfield EH 1994 Pituitary magnetic resonance imaging in normalhumanvolunteers: occult adenomas in the general population. Annals of Internal Medicine 120 817-820.

6 Ilias I, Torpy DJ, Pacak K, Mullen N, Wesley RA \& Nieman LK 2005 Cushing's syndrome due to ectopic corticotropin secretion: twenty years' experience at the National Institutes of Health. Journal of Clinical Endocrinology and Metabolism 90 4955-4962. (doi:10.1210/ jc.2004-2527)

7 Lynnette KN, Beverly MKB, James WF, Murad MH, John N-P, Martin OS \& Antoine T 2015 Treatment of Cushing's syndrome: an endocrine society clinical practice guideline. Journal of Clinical Endocrinology and Metabolism 100 2807-2831. (doi:10.1210/jc.2015-1818)

8 Newell-Price J, Trainer P, Besser M \& Grossman A 1998 The diagnosis and differential diagnosis of Cushing's syndrome and pseudo-Cushing's states. Endocrine Reviews 19 647-672. (doi:10.1210/er.19.5.647)

9 Susmeeta TS \& Lynnette KN 2011 Cushing's syndrome: all variants, detection, and treatment. Endocrinology and Metabolism Clinics of North America 40 379-391. (doi:10.1016/j.ecl.2011.01.006)

10 Graham BS \& Tucker WS 1984 Opportunistic infections in endogenous Cushing's syndrome. Annals of Internal Medicine 101 334-338.

11 Gilbert GF \& Rena V-S 2007 Hypercortisolemia and infection. Infectious Disease Clinics of North America 21 639-657.

12 van der Pas R, Leebeek FW, Hofland LJ, de Herder WW \& Feelders RA 2013 Hypercoagulability in Cushing's syndrome: prevalence, pathogenesis and treatment. Clinical Endocrinology 78 481-488. (doi:10.1111/cen.12094)

Received in final form 17 August 2017

Accepted 31 August 2017 